\title{
Levodopa:
}

\section{the story so far}

\section{Alison Abbott explores the history of the first treatment for Parkinson's disease since its dramatic debut in the swinging sixties.}

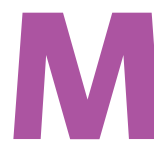

ore than four decades after his discovery that dopamine was a neurotransmitter and could control movement, Swedish pharmacologist Arvid Carlsson won a share of the 2000 Nobel Prize in Physiology or Medicine. His discovery had led swiftly and directly to the development of levodopa (L-DOPA) - a precursor of dopamine that passes through the blood-brain barrier - as a revolutionary treatment for Parkinson's disease.

L-DOPA burst onto the clinical scene in the late 1960s as a miracle drug, unlocking or awakening patients immobilized for years or decades by the crippling and progressive neurodegenerative disease. Its debilitating side effects became apparent with equal swiftness. These include disturbing dyskinesias or uncontrolled movements and equally disturbing on-off effects in which the effect of the drug suddenly stops, freezing patients almost mid-sentence.

But L-DOPA turned out to be a most difficult molecule to control therapeutically, thanks to its inconveniently short biological half-life, physicochemical cussedness and poorly understood pharmacodynamic interaction with disease progression. These problems have not been overcome, although there are some promising approaches to L-DOPA delivery in the pipeline. Neurologists still fight to find the balance between clinical effectiveness and side effects in individual patients. For all that, L-DOPA remains the gold-standard treatment, and many experts assert that life expectancy on current treatment regimens is close to that of the normal population.

\section{Against the tide}

Shaking the hand of his country's king was one of the most joyous occasions of Carlsson's long life. But in his acceptance lecture he recalled the hostility that greeted him when he presented the results of his key experiments in the late 1950s. The powerful European pharmacological community was locked into a mindset that held dopamine to be no more than a precursor in the biosynthesis of noradrenaline (Fig. 1), with no significant biological activity of its own.
D,L-DOPA was first synthesized in 1911 by Casimir Funk, the Polish biochemist who coined the term vitamin. In 1913, Marcus Guggenheim, a biochemist from Hoffmannla Roche in Basel, isolated the pure enantiomer L-DOPA from the exotic bean plant Vicia faba. Back then, no-one saw reason to patent a molecule that appeared to have no useful biological activity. Indeed, it was seen as toxic. Guggenheim vomited violently when he tried it on himself - because, as later became apparent, L-DOPA is converted to dopamine, an emetic, in the body.

Carlsson made his dramatic discovery while researching an interesting new drug called reserpine, introduced in the mid-1950s as the first selective treatment for schizophrenia. In fact, reserpine had severe side effects, including a tendency to induce parkinsonian symptoms, and was quickly replaced by safer drugs.

Pharmacologists discovered that reserpine depleted levels of the neurotransmitter serotonin in the brain, and that rabbits injected with it become instantly catatonic. Carlsson, fresh from an inspiring research visit to the United States and in the throes of equipping his first laboratory, bought an expensive, state-ofthe-art item he considered indispensible for carrying the research forward: a spectrophotofluorimeter able to measure chemically the levels of neurotransmitters synthesized from fluorescently-tagged precursors. With this, he showed that reserpine caused depletion not just of the neurotransmitter serotonin, but also of the chemically similar agents noradrenaline and adrenaline.

Which of these neurotransmitters would reverse the catatonia of the rabbits? Carlsson decided to inject the animals with L-DOPA because neither noradrenaline nor dopamine cross the blood-brain barrier. Within 15 minutes of the injection, the rabbits were hopping around as if nothing had happened. He found that 5-hydroxytryptophan, the precursor of serotonin, did not reverse the catatonia.

At the 1960 Ciba Foundation Symposium on Adrenergic Mechanisms in London, Carlsson presented a paper proposing dopamine as a neurotransmitter and implicating it in Parkinson's disease. He was shocked by the reception. His concepts were wholeheartedly rejected by a traditional community used to measuring the activity of neurotransmitters with biological assays. Chemical assays with Carlsson's spectrophotofluorimeter did not impress them.

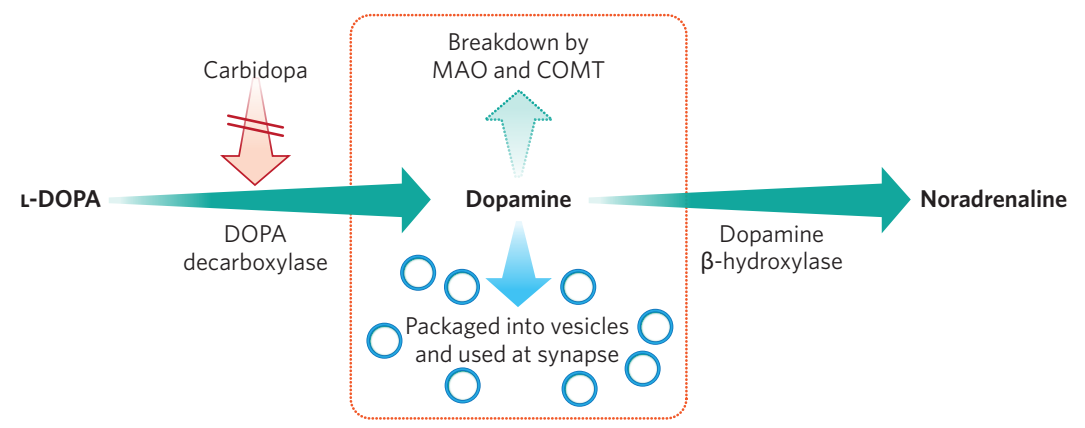

Figure 1 | Dopamine synthesis and activity in neurons. Enzymes (that can be blocked by carbidopa) turn L-DOPA into dopamine. It is then either used, broken down or further transformed into noradrenaline.

Still, his publications were read and understood by many. A young Austrian scientist, Oleh Hornykeiwicz, decided to look at dopamine levels in post-mortem brains of Parkinson's disease patients. He found them entirely depleted in an area called the striatum. Reasoning that symptoms could be caused by lack of dopamine and thus reversed by replacing it, he joined forces, in 1961, with neurologist Walter Birkmayer to inject L-DOPA into 20 severely parkinsonian patients. This achieved miraculous, if temporary, effects: for a few hours, their rigid limbs melted into movement.

Building on Hornykeiwicz's work, a few years later neurologist George Cotzias, at the Brookhaven National Laboratory on Long Island, New York, began trials of an oral form of L-DOPA. His 1968 New England Journal of Medicine paper, reporting a successful twoyear study on 28 patients, made the pages of Time magazine. The US Food and Drug Administration approved the drug in 1970. 


\section{Taming levodopa}

Since then, L-DOPA has been the mainstay of Parkinson's disease therapy. Remarkably, no more efficacious drug has yet been developed, although there have been useful attempts. Dopaminergic agonists, which mimic dopamine at its receptors, provide invaluable therapeutic support, says Kieran Breen, director of research at Parkinson's UK, a support and research charity, "but they are less effective and have their own side effects." Around $14 \%$ of patients on dopamine agonists develop impulse-control disorders, turning some into gamblers.

With L-DOPA, a good two-thirds of patients develop mild or severe dyskinesias following several years of therapy, after which the disturbing on-off phenomenon also kicks in. Ideally, L-DOPA would be packaged and delivered in a form that avoided these side effects, but the molecule turned out to be a pharmacologist's nightmare. It looks small and innocent, but has an incompliant chemistry and unhelpful metabolism.

For one thing, it is removed rapidly by enzymes in the blood where it has a half-life of around an hour. It was relatively easy to improve on this by judicious use of enzyme inhibitors. L-DOPA is converted to dopamine by the aromatic amino-acid decarboxylase enzyme in the blood. This source of dopamine causes peripheral side effects like nausea and reduces the amount of L-DOPA available to cross into the brain. Since the late 1970s, L-DOPA has been given together with carbidopa, or another inhibitor of this enzyme, stretching its half-life in the blood to 90 minutes. More recently, clinicians have taken to adding inhibitors of dopamine's two

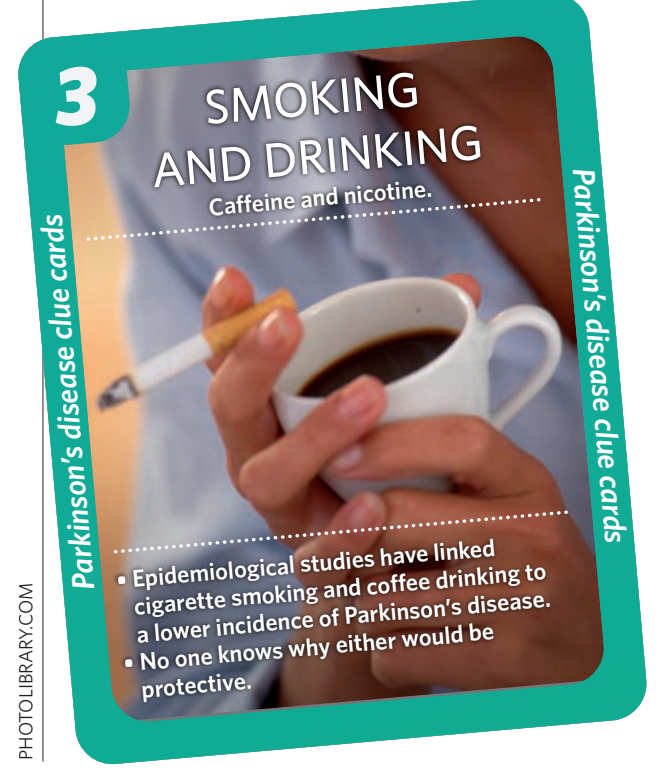

breakdown enzymes, catechol-O-methyltransferase (COMT) and monoamine oxidase (MAO), to the therapeutic mix, extending the duration of L-DOPA's effects further.

This modestly extended half-life is of little help when it comes to problems after longterm use. In the brain, L-DOPA is handled as if it were naturally produced. It is taken up into dopaminergic nerve terminals where it is briefly stored before being converted into dopamine Arvid Carlsson (left) receives the Nobel prize for his work on dopamine and released upon nerve from the Swedish king Carl XVI Gustaf (right) in 2000. stimulation. The nerve terminals thus provide a short-term buffering of the variable blood levels, allowing the drug effect to be maintained between doses.

As the disease progresses and more dopaminergic nerve terminals are destroyed, however, this buffering capacity shrinks, and the therapeutic effect of L-DOPA starts to reflect its blood levels. Why this should promote dyskinesias is not certain, but a prevailing theory holds that it is because the dopamine-depleted brain becomes more sensitive to external dopamine in compensation and thus overreacts to the dopamine generated from L-DOPA. The on-off effects are also not entirely understood because the off times do not always coincide with low blood levels of L-DOPA.

What is clear is that stabilizing the blood levels greatly ameliorates these undesirable effects. This was shown back in the 1980s by Maral Mouradian, now at the University of Medicine and Dentistry, New Jersey-Robert Wood Johnson Medical School, in trials in which patients with extreme symptoms were placed on continual intravenous infusion of L-DOPA. However, slowly dripping the drug into the blood $24 / 7$ "is clearly not a practical solution", says Mouradian. And not just because no-one can comfortably live attached to a drip; L-DOPA is also poorly soluble in water so the quantities needed for therapeutic effect are enormous, and it irritates veins and soft tissues.

For maximum convenience, L-DOPA would be delivered via a device such as a skin patch. Once again, however, L-DOPA's physiochemical properties let it down: it does not pass easily through the skin. As yet, no patch is on the market.

Most alternative approaches to oral delivery - like nasal or rectal — have also failed.
And sustained-release oral preparations of L-DOPA have so far disappointed. L-DOPA is absorbed in an inconveniently short stretch of the intestine - the duodenum and upper jejunum, just below the stomach — limiting the time available for any formulation. Absorption is also limited by the regularity with which the stomach empties into the intestine, and this can be erratic in Parkinson's disease patients. Many ingenious approaches are being tried to get around these problems, like attaching L-DOPA to materials that are actively retained in the stomach and letting it seep slowly into the duodenum. A system of microtablets is also under development, comprising tiny L-DOPA pills in a special dispenser, to allow more frequent but smaller doses according to individual need.

One solution for continuous dopaminergic stimulation is the so-called Duodopa system. It was approved for use in advanced Parkinson's disease in several European countries in 2004 and is currently being fast tracked by the US Food and Drug Administration. Duodopa is a microsuspension gel of L-DOPA and carbidopa that is pumped directly into the duodenum via a surgically implanted cannula. However, the treatment is invasive and and expensive, costing around \$35,000 per year, says Mouradian.

That no better drug for Parkinson's disease has been found than the first one discovered 40 years ago is a vanishingly rare pharmacological phenomenon. L-DOPA does not slow the inexorable progress of the disease, but if a better way could be found to deliver it then it could keep patients mobile for longer - and delay the need for more radical intervention, such as deep brain stimulation.

Alison Abbott is Nature's Senior European Correspondent. 